\title{
IOT based Indoor Air Pollution Monitoring using Raspberry PI
}

\author{
Dr.B.Sivasankari \\ Associate professor, Department of Electronics and Communication Engineering \\ SNS College of technology, Coimbatore, Tamil Nadu, India \\ C.Arun prabha, S.Dharini ,R.Haripriya \\ UG Students, Department of Electronics and Communication Engineering \\ SNS College of technology, Coimbatore, Tamil Nadu, India
}

\begin{abstract}
This paper proposes an approach to monitor Air pollution parameter using the Raspberry-Pi.The system is designed using the python coding language. The monitored values can be accessed from the Internet of Things platform. The air pollution parameters are taken from the low cost gas sensors. The parameters include: concentrations of smoke, carbon monoxide and nitrogen-di-oxide, temperature and humidity. Also an alarm is triggered to indicate high concentrations of emissions. This acts as a warning to the authorities about the air pollution rate. A graph is plotted using the monitored values using Thing speak platform.
\end{abstract}

Keywords - Raspberry pi, Internet of things, Thing speak platform, python coding, gas sensors.

\section{INTRODUCTION}

Air pollution means presence of high concentrations of harmful gases such as dust, smoke. Inhaling these gases can increase the chances of health problem .In fact, dust when inhaled can cause breathing problems, damage lung tissue, and boost up existing health problems. Greenhouse gases trap heat and make the earth warmer. Human activities are responsible for almost all of the increases in greenhouse gases. Therefore, every federal government has stringent regulations which require prevention and reduction of emission levels. In our project, the major air pollutants like $\mathrm{CO} 2, \mathrm{NO} 2, \mathrm{CO}$ is monitored using sensors and values obtained are processed using Raspberry Pi. Then the data's are sent to an IP address and can be monitored from anywhere from logging into the IP address

The rest of the paper is organized as follows: Literature survey is explained in section II, Existing system in section III, proposed system in section IV, Results and discussion in section V and Conclusion in section VI.

\section{LITERATURE SURVEY}

[1] Air quality monitoring systems that can monitor gas such as CO and Sox on ambient air in a real-time and can be accessed with internet line have been developed. Sensor element on this system is based on Nano structured zinc oxide thin film synthesized using wet chemical route. Monitoring system is designed using arduino Uno microcontroller as analog to digital converter, and ethernet shield for data transmission, computer server for database center and data acquisition. The data from this monitoring system can be accessed and viewed as web-page. The sensor element that used in this system is made at nanostructure so it will yield high sensitivity. The optimum experimental parameters that will be used are temperature, exposure time to gas target, sampling period, and also ratio for regeneration time. Measurements will be held under well-controlled and artificially $\mathrm{CO} / \mathrm{Sox}$ polluted atmosphere.

[2] Due to the increasing industrialization and the massive urbanization, air pollution monitoring is being considered as one of the major challenges of smart cities. Many air pollution monitoring systems have been proposed in the literature, among which wireless sensor networks seem to be a leading solution. A careful deployment of sensors is therefore necessary to get better Performances while ensuring a minimal financial cost. In this paper, the citywide wireless sensor networks are considered and tackle the minimum-cost node positioning issue for air pollution monitoring. The proposed system has an efficient approach that aims to find optimal sensors and sinks locations while ensuring air pollution coverage and network connectivity. Unlike most of the existing methods, 
which rely on simple and generic detection models, our approach is based on the spatial analysis of pollution data, allowing to take into account the nature of the pollution phenomenon.

[3] A Wi-Fi based plug and sense smart device for dedicated air pollution monitoring using Internet of Things is designed. This system designed on device to cloud architecture in IoT for monitoring air pollution precisely. Once the sensor node reads individual pollutants composition and location coordinates, Air quality index (AQI) will be calculated using linear segmented principle with greater Vancouver AQI table and Max operator aggregation method. Based on AQI value, corresponding LED will be actuated for indication and health impact with precaution steps messages will be displayed on the screen. All those data will be pushed to thing speak cloud storage, an open source application Programming interface for IoT based devices. These pushed data along with Date and time can be retrieved as a separate excel sheet for future analysis. Through thing view android app, real time pollution level with location can be visualized in terms of line graph. With the implementation of this low cost and small size smart device, alert can be given to people to wear anti-pollution mask and reroute path in transportation where there is high air pollution ensuring high reliability and consistency.

\section{EXISTING SYSTEM}

\section{A.AIR POLLUTION MONITORING USING ARDUINO UNO BOARD:}

The commercial meters available in the market such as Fluke C0-220, Carbon monoxide meter for CO, am probe $\mathrm{CO} 2$ meter for $\mathrm{CO} 2$, Forbix Semicon LPG gas leakage sensor alarm for LPG leakage detection have been used in before cases.

The existing system is build using the Arduino Uno board and output is shown in the LCD display. Arduino board is not full computers and also they are designed for specific purpose. They don't have an in build Wi-Fi port. They don't run a full operating system, but simply execute the written code.

\section{B.DISADVANTAGES OF USING ARDUINO UNO BOARD:}

The arduino isn't built for network connectivity directly. It requires a bit more tinkering to set up a proper connection, though it is possible. There is a need of extra chip with an ethernet port and it must be coded to wire with the arduino board. This introduces the disadvantage that it requires extra hardware. The raspberry pi comes ready with an inbuilt Wi-Fi port which makes the system easier for IOT applications. This is one of the reasons why the pi is the device of choice for things like personal webservers, printer servers and VPN's. The clock speed in arduino is low compared to Raspberry pi that is 40 times slower than pi.The RAM of raspberry pi is 12,800 times larger compared to arduino. This is the reason for switching from arduino to raspberry pi since they have more advantage for IOT applications.

\section{C.ESP8266 WITH ARDUINO:}

In order to connect a Wi-Fi module with arduino, there is a need of extra chip.ESP8266 is the most commonly used chip for Wi-Fi connectivity. The chip must be connected separately. This chip is not capable of 5-3 logic shifting and requires an external convertor. It also has disadvantages that there is no access to all the input and output pins of this chip and problems occur for complex projects.

The arduino libraries are not very efficient in certain parts and waste RAM and CPU cycles and also limited to small number of MCU's. Also arduino is only officially supported on Atmel AVR and Atmel SAM series. There are ports and adaptions to a few other MCU's like ESP2866 or the NRF51 series, but they don't seem to be fully supported.

\section{PROPOSED SYSTEM}

In the proposed system, we are going to make an IOT based air pollution monitoring system using raspberry pi.

A.RASPBERRY PI

Volume 9 Issue 2 December 2017 
This project is build using raspberry pi 3b.The pi has a Broadcom BCM2835 system on a chip (SOC) which includes an ARM1176JZF-S $700 \mathrm{MHz}$ processor. It does not include a built-in hard disk or solid state drive, but it uses an SD card for booting and persistent storage. Also the pi is does not come shipped with an operating system. Tools are available for python coding as the main language. The pi can also be coded with c, Perl, java languages.

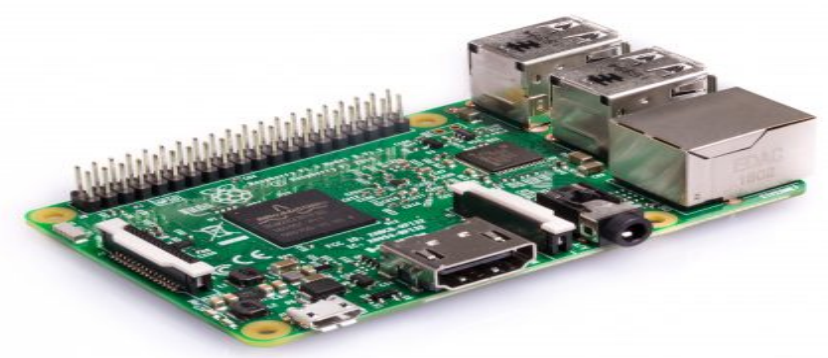

Figure.1 Raspberry pi 3b

These pins are a physical interface between the pi and the outside world. At the simplest level, out of 40 pins, 20 pins are GPIO pins and the others are power or ground pins.

GPIO Pinout Diagram

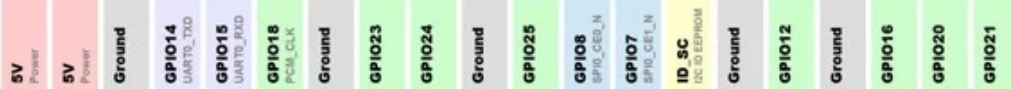

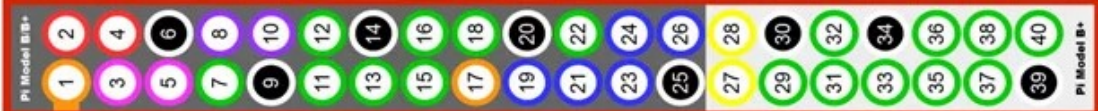

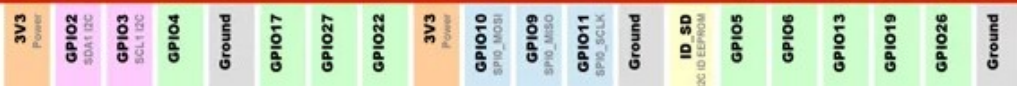

Figure.2 Raspberry pi GPIO pinout diagram

C.BLOCK DIAGRAM OF PROPOSED SYSTEM: 


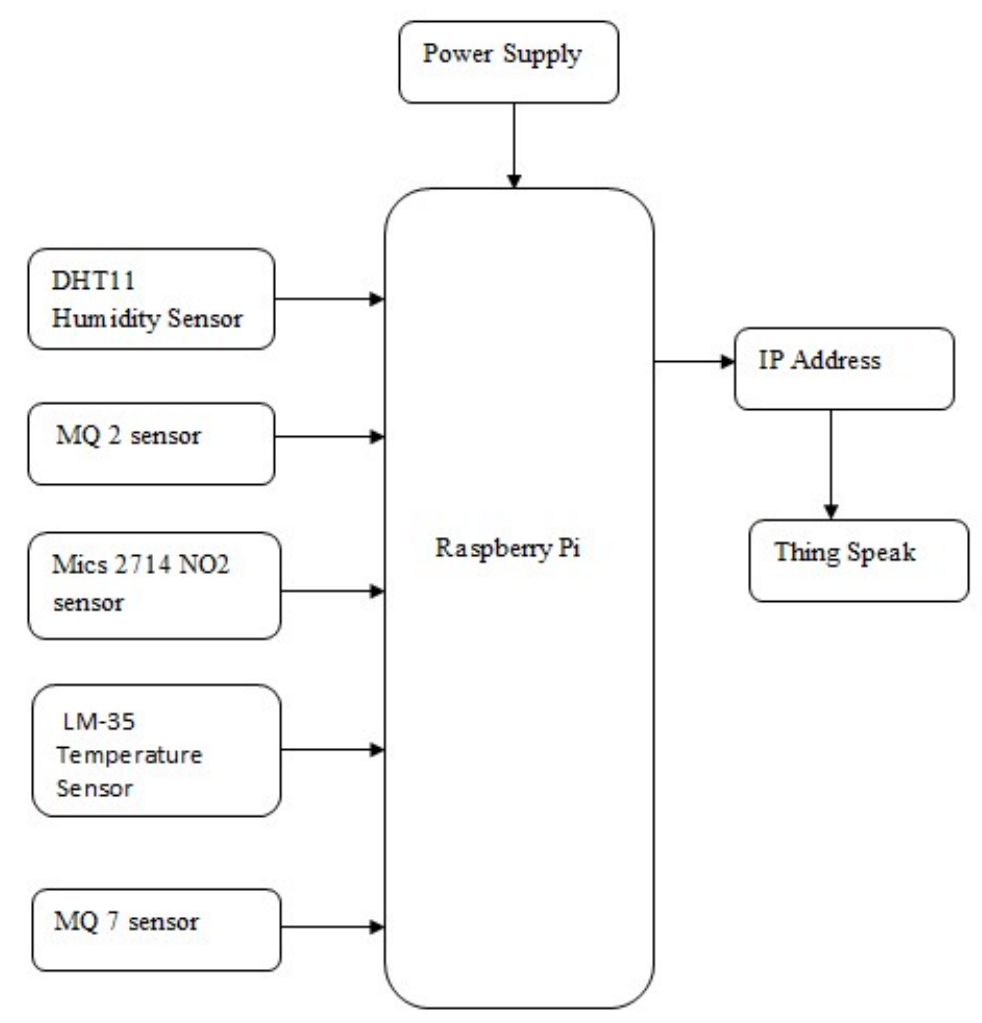

Figure. 3 block diagram of proposed system

\section{D.SENSORS USED}

1) Humidity sensor:

The DHT11 Sensor is used for humidity measurement. It is fairly simple to use but requires careful timing to grab data. The new reading is obtained every 2 seconds. This sensor is good for $20-80 \%$ humidity readings with $5 \%$ accuracy.It have 4 pins with 0.1 spacing.

2) C02 gas sensor:

This is an ideal sensor to detect the presence of dangerous LPG gas concentrations. This unit can be easily incorporated into an alarm unit. This sensor can also indicate LNG, propane, butane and cigarette smoke .It has high sensitivity with detection range 100-10000 ppm.

3) MICS 2714 sensor:

This sensor's resistance varies even with small concentrations of NO2.The resistance varies between $2 \mathrm{~K} \Omega$ and $2 \mathrm{M} \Omega$.It has air resistance of 0.8 to 8 kilo ohms. The sensitivity ranges from 6 to $100 \mathrm{ppm}$ and the response time is 30 seconds.

4) $M Q 7$ sensor:

This sensor is used for sensing CO concentrations. It has high sensitivity and fast response time. It gives values in the range of 20 to 2000 ppm.It requires power supply of $5 \mathrm{v}$.

5) LM35 sensor: 
LM 35 sensor is a precision temperature sensor with its output proportional to the temperature. With this sensor, temperature can be measured more accurately than with a thermistor. It also possesses low self-heating and does not cause more than $0.1^{\circ} \mathrm{C}$. Temperature rise in still air. The operating temperature range is $-55^{\circ} \mathrm{C}$ to $150^{\circ} \mathrm{C}$.

D) $M C P 3008 A D C$ :

This is a low cost Analog to digital convertor. Since raspberry pi do not have analog pins, analog to digital conversion is done. It has 8 channels and so 8 sensors can be interfaced.

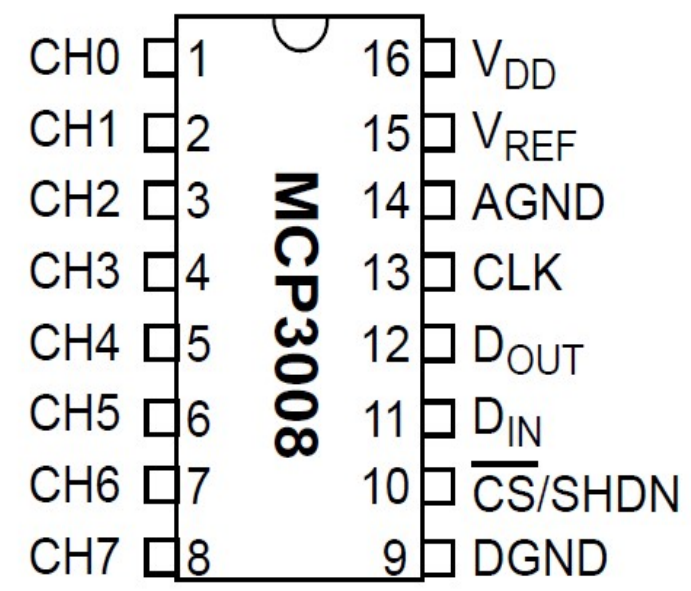

Figure.4 MCP3008 PIN diagram

\section{RESULTS AND DISCUISSION}

The MQ2 gas sensor has been interfaced with the raspberry pi using the python coding language and output is measured using the thinner sample and shown in the shell widow. The values measured are in ppm (parts per million) range. Similarly other sensors can be interfaced with raspberry pi. 


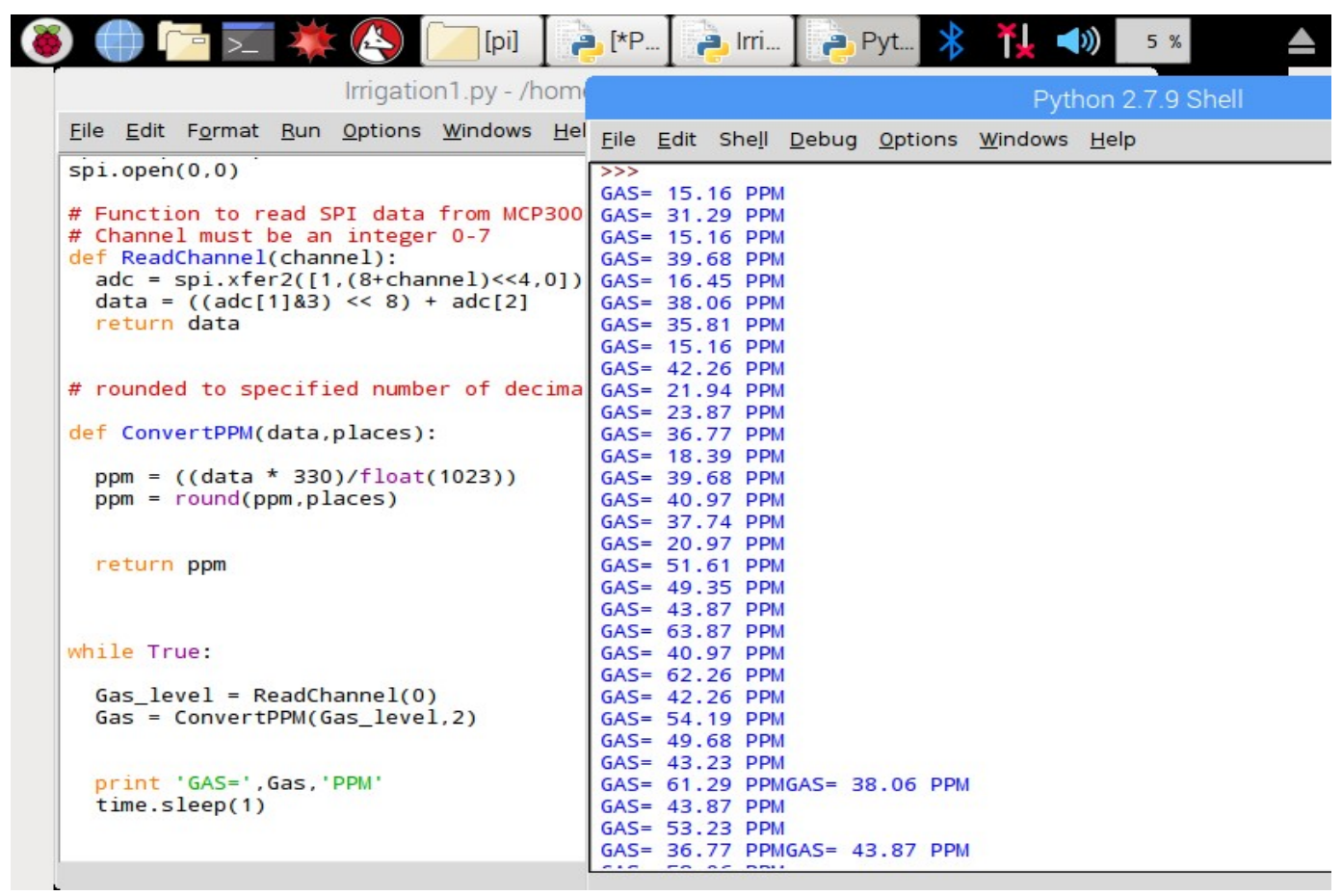

Figure.5 MQ2 sensor with raspberry pi

\section{CONCLUSION}

The sensors when interfaced with raspberry pi gives out values in ppm (parts per million). The threshold value can be fixed accordingly to trigger out alarm. These data are sent to an IP address and also graph can be plotted for every half-an-hour to indicate the concentrations of the pollutants using the thing speak platform.

\section{REFERENCES}

[1] Pope III, C. A., Burnett, R. T., Thun, M. J., Calle, E. E., Krewski, D., Ito, K., \& Thurston, G. D., "Lung cancer, cardiopulmonary mortality, and long-term exposure to fine particulate air pollution," Journal of the American Medical Association, 287(9), pp. 1132-1141.

[2] George F. Fine, Leon M. Cavanagh, Ayo Afonja and Russell Binion, "Metal Oxide Semi-Conductor Gas Sensors in Environmental Monitoring," Sensors 2010, Vol. 10, pp. 5469-5502, 2010.

[3] Duk-Dong Lee and Dae-Sik Lee, "Environmental Gas Sensors," IEEE Sensors Journal, Vol. 1, No. 3, pp.

214-224, Oct. 2001.

[4] WHO, "WHO guidelines for indoor air quality: selected pollutants," WHO, 2010.

[5] Gomez, Carles, Joaquim Oller, and Joseph Paradells, "Overview and evaluation of Bluetooth low energy: An emerging low-power wireless technology," Sensors 12.9 (2012), pp. 11734-11753.

[6] Thread Group, "Introducing Thread: A New Wireless Networking Protocol for the Home, "http://www.threadgroup.org, 2014.

[7] Shelby, Zach, and Carsten Bormann, "6LoWPAN: the wireless embedded internet," Vol. 43. John Wiley \& Sons, 2011.

[8] IEEE 802.15 WPANTM, Ultra Low Power Task Group 15.4q Ultra Low Power (ULP) TG4q (Technical Guidance Document Draft, 2015. 